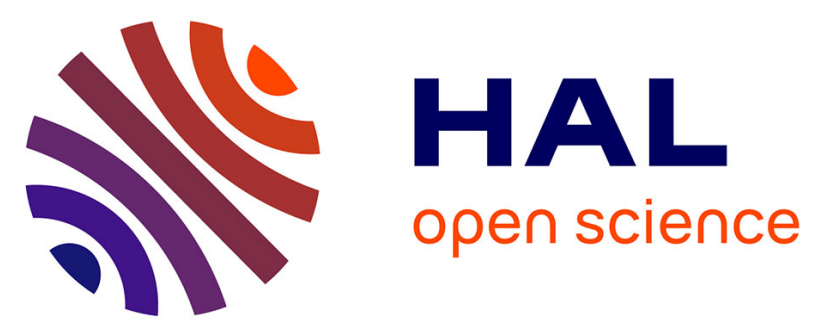

\title{
Channel estimation with a priori position for aeronautical communications via a satellite link
}

Bilel Raddadi, Charly Poulliat, Nathalie Thomas, Marie-Laure Boucheret, Benjamin Gadat

\section{- To cite this version:}

Bilel Raddadi, Charly Poulliat, Nathalie Thomas, Marie-Laure Boucheret, Benjamin Gadat. Channel estimation with a priori position for aeronautical communications via a satellite link. 26th IEEE International Conference on Personal, Indoor and Mobile Radio Communications (PIMRC 2015), Aug 2015, Hong Kong, Hong Kong SAR China. pp. 532-537. hal-01461704

\author{
HAL Id: hal-01461704 \\ https://hal.science/hal-01461704
}

Submitted on 8 Feb 2017

HAL is a multi-disciplinary open access archive for the deposit and dissemination of scientific research documents, whether they are published or not. The documents may come from teaching and research institutions in France or abroad, or from public or private research centers.
L'archive ouverte pluridisciplinaire HAL, est destinée au dépôt et à la diffusion de documents scientifiques de niveau recherche, publiés ou non, émanant des établissements d'enseignement et de recherche français ou étrangers, des laboratoires publics ou privés. 


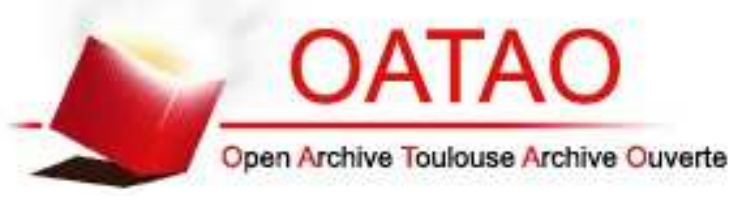

\section{Open Archive TOULOUSE Archive Ouverte (OATAO)}

OATAO is an open access repository that collects the work of Toulouse researchers and makes it freely available over the web where possible.

This is an author-deposited version published in : http://oatao.univ-toulouse.fr/ Eprints ID : 17193

The contribution was presented at PIMRC 2015 :

https://www.ieee.org/conferences_events/conferences/conferencedetails/index.html? Conf_ID $=35684 \& W T . m c \_i d=u e m \_26 a$

To cite this version : Raddadi, Bilel and Poulliat, Charly and Thomas, Nathalie and Boucheret, Marie-Laure and Gadat, Benjamin Channel estimation with a priori position for aeronautical communications via a satellite link. (2015) In: 26th IEEE International Conference on Personal, Indoor and Mobile Radio Communications (PIMRC 2015), 30 August 2015 - 2 September 2015 (Hong Kong, Hong Kong).

Any correspondence concerning this service should be sent to the repository administrator: staff-oatao@listes-diff.inp-toulouse.fr 


\section{Channel estimation with a priori position for aeronautical communications via a satellite link.}

\author{
Bilel RADDADI, Charly POULLIAT, \\ Nathalie THOMAS, Marie-Laure BOUCHERET \\ University of Toulouse, INPT/ENSEEIHT, IRIT, France \\ \{vorname.name\}@enseeiht.fr
}

\author{
Benjamin GADAT \\ Thales Alenia Space, Toulouse, France \\ benjamin.gadat@thalesaleniaspace.com
}

\begin{abstract}
In this paper, we investigate on some efficient channel estimation methods for the aeronautical multipaths channel. The proposed methods exploit both the particular form of the channel impulse response and a priori knowledge of some parameters (mainly delays) that can be inferred from geometrical considerations based on geolocation. The first estimation method is based on a parametric multipath channel model while the second tries to exploit the relative sparsity of the channel impulse response. In both cases, this reduces the number of variables to be estimated and it provides better performance compared to a direct classical least-square estimation of the discrete equivalent channel impulse response.
\end{abstract}

\section{INTRODUCTION}

In wireless communication systems, the estimate of the channel impulse response is necessary for the coherent demodulation. For this, it is common to introduce training sequences in the transmitted signal [1]. If the channel varies over time and if one knows the coherence time, one can then define the frames so that the channel can be assumed to be stationary on each. When estimating directly the equivalent discrete baseband channel impulse response, the use of the maximum likelihood (ML) criterion leads to an optimal solution. However, this estimation process can be very complex due to the memory channel which is generally long. In the presence of Gaussian noise, ML estimation becomes equivalent to the least square criterion (LS)[2] and reaches the CramerRao bound (CRB).Under some considerations, improvements can be achieved if one is able to perform joint estimation of both attenuations and delays of the continuous impulse response which is generally practically achieved through an iterative estimation procedure [2]. These methods are based on a explicit multipath parametric model. However, in some transmission systems, such as aeronautical communication systems, some parameters for the continuous channel can be determined in a very accurate manner, such as the delays of the different paths, based on some geometrical considerations from geolocation techniques [3]. This extra a priori allows us to further reduce the unknowns of our system and leads to more efficient multipath parametric based estimation methods. Only the attenuation coefficients of the multipath channel will be estimated. This has to be compared with the classical estimation of all taps of the discrete equivalent channel as usually done. Other features could be exploited such as the sparsity of the channel [4].
In this paper, we are interested in the estimation of the aeronautical multipath channel. Based on parametric models for the impulse response of the multipath channel, we propose simple but efficient channel estimation methods that benefit from a known position of the mobile user. The proposed methods exploit both the particular form of the channel impulse response and a priori knowledge of some parameters (mainly delays) that can be inferred from geometrical considerations based on geolocation. The first estimation method is based on a parametric multipath channel model while the second method tries to exploit the relative sparsity of the channel impulse response. In both cases, this reduces the number of variables to be estimated and it provides better performance compared to a direct classical least-square estimation of the discrete equivalent channel impulse response.

The paper is organized as follows. Section II reviews conventional channel estimation methods using the least square criterion (LS) with and without using a parametric multipath model. Section III provides an application to aeronautical communications via a satellite link and describes the two proposed estimation methods. Simulation results are presented in Section IV, while Section V concludes the article.

\section{Position BASED CHANNEL ESTIMATION: A MULTI-PATH PARAMETRIC APPROACH}

\section{A. System model}

We consider a linear multipath channel with single input and single output (SISO), that is time-invariant, where $\left\{h_{l}, 0 \leq\right.$ $l \leq L-1\}$ is the equivalent channel impulse. Assuming that the transmit and receive filters $h_{e}(t)$ and $h_{r}(t)$ are both Root Raised Cosine (RRC) filters with known parameters, the impulse response of the global equivalent channel is given by:

$$
h(t)=\sum_{n=0}^{N_{t}-1} a_{n} p\left(t-\tau_{n}\right)
$$

where, $a_{n} \in \mathbb{C}$ and $\tau_{n}$ are respectively the attenuation and the delay of the $n^{\text {th }}$ path and $N_{t}$ is the number of paths.

After sampling at the symbol period $T_{s}$, the discrete equivalent impulse response channel is given by:

$$
h_{l}=h\left(l T_{s}\right)=\sum_{n=0}^{N_{t}-1} a_{n} p\left(l T_{s}-\tau_{n}\right) \quad 0 \leq l \leq L-1
$$


where $p(t)=h_{e}(t) * h_{r}(t)$.

We use a $N_{p}$ training sequence $\boldsymbol{x}=\left(x_{0}, x_{1}, \ldots, x_{N_{p}-1}\right)$, consisting of pilot symbols $x_{i}, i=0, \ldots, N_{p}-1$ with symbol power $\sigma_{p}^{2}$ and $N_{p} \geq L$. Finally, the received signal can be written as:

$$
y_{k}=\sum_{l=0}^{L-1} h_{l} x_{k-l}+w_{k}
$$

, where $w_{k}, k=0 \cdots N_{p}-1$, are independent and identically distributed additive white Gaussian (AWGN) noise samples with variance $\sigma_{w}^{2}$. The discrete channel impulse response $\boldsymbol{h}=$ $\left(h_{0}, h_{1}, \ldots, h_{L-1}\right)^{T}$ will be assumed constant over a frame duration. Its memory $L$ is supposed equal to the memory of the raised-cosine filter $p(t)$ and it is perfectly known at the receiver side.

\section{B. Unstructured LS estimator U-LSE}

Unstructured LS estimation denotes the direct estimation of the discrete equivalent channel $\boldsymbol{h}=\left(h_{0}, h_{1}, \ldots, h_{L-1}\right)^{T}$ without any a priori. For ease of presentation, we review here the main results of a direct estimation of $\boldsymbol{h}$.

Assuming that $\boldsymbol{h}=\left(h_{0}, h_{1}, \ldots, h_{L-1}\right)^{T}$ is constant over one transmission frame and unknown, the equation (3) can be written using matrix notations as follows:

$$
\boldsymbol{y}=\boldsymbol{X} \boldsymbol{h}+\boldsymbol{w}
$$

where

$$
\boldsymbol{X}=\left(\begin{array}{cccc}
x_{L-1} & x_{L-2} & \ldots & x_{0} \\
x_{L} & x_{L-1} & \ldots & x_{1} \\
x_{L+1} & x_{L} & \ldots & x_{2} \\
\vdots & \vdots & \ldots & \vdots \\
x_{N_{p}-1} & x_{N_{p}-2} & \ldots & x_{N_{p}-L}
\end{array}\right)
$$

and $\boldsymbol{y}=\left[y_{L-1}, y_{L}, \ldots, y_{N_{p}-1}\right]^{T}, \quad \boldsymbol{w}=$ $\left[w_{L-1}, w_{L}, \ldots, w_{N_{p}-1}\right]^{T}$. The corresponding Cramer-
Rao bound (CRB) for the estimation of $\boldsymbol{h}$, noted $\boldsymbol{C} \boldsymbol{R} \boldsymbol{B}_{U}(\boldsymbol{h})$ can be obtained as follow:

$$
C R B_{U}(h)=F^{-1}(h)
$$

where $F(\boldsymbol{h})$ is the Fisher information matrix given by:

$$
\begin{aligned}
\boldsymbol{F}(\boldsymbol{h}) & =\mathbb{E}\left\{\boldsymbol{\nabla}_{\boldsymbol{h}} \log (p(\boldsymbol{y}, \boldsymbol{h}))\left[\boldsymbol{\nabla}_{\boldsymbol{h}} \log (p(\boldsymbol{y}, \boldsymbol{h}))\right]^{H}\right\} \\
& =\frac{1}{\sigma_{w}^{2}}\left(\boldsymbol{X}^{H} \boldsymbol{X}\right)
\end{aligned}
$$

$\nabla_{h}$ denotes the gradient operator according to the vector $\boldsymbol{h}$ and $f(\boldsymbol{y} / \boldsymbol{a})=\frac{1}{\left(\sqrt{\pi \sigma_{w}^{2}}\right)^{N_{p}-L+1}} \exp \left(-\frac{1}{\sigma_{w}^{2}}\|\boldsymbol{y}-\boldsymbol{X} \boldsymbol{h}\|_{2}^{2}\right)$. An estimation according to the maximum likelihood criterion (ML) can reach the CRB in the presence of AWGN noise. In that case the unbiased ML estimator $\hat{h}_{M L}$ is exactly the one given by the unbiased least-square (LS) solution $\hat{h}_{U-\boldsymbol{L} S}$ given by:

$$
\hat{\boldsymbol{h}}_{\boldsymbol{U}-\boldsymbol{L} \boldsymbol{S}}=\left(\boldsymbol{X}^{H} \boldsymbol{X}\right)^{-1} \boldsymbol{X}^{H} \boldsymbol{y}
$$

Based on $C R B_{U}(h)$, a lower bound for the Mean Square Error (MSE) $\boldsymbol{M S E}\left(\hat{\boldsymbol{h}}_{\boldsymbol{U}-\boldsymbol{L} \boldsymbol{S}}\right)=\mathbb{E}\left\{\left\|\hat{\boldsymbol{h}}_{\boldsymbol{U}-\boldsymbol{L} \boldsymbol{S}}-\boldsymbol{h}\right\|_{2}^{2}\right\}$ is classically given as:

$$
\boldsymbol{M S E}\left(\hat{\boldsymbol{h}}_{\boldsymbol{U}-\boldsymbol{L} \boldsymbol{S}}\right)=\operatorname{tr}\left\{\boldsymbol{C R} \boldsymbol{B}_{\boldsymbol{U}}(\boldsymbol{h})\right\}=\sigma_{w}^{2} \operatorname{tr}\left\{\left(\boldsymbol{X}^{H} \boldsymbol{X}\right)^{-1}\right\}
$$

\section{The Multipath Parametric Model: Structured LS estima-} tor $S$-LSE

The preceding approach does not take into account any prior information on the propagation model. This approach can be further improved by considering a parametric model and applying joint estimation of the attenuations and the multipath delays [2]. Thus, if we now write our model to introduce a parametric dependence on the propagation delays equation, equation(1) can be expressed as:

$$
\boldsymbol{h}=\boldsymbol{P}(\boldsymbol{\tau}) \boldsymbol{a}
$$

where $\boldsymbol{a}=\left(a_{0}, \ldots, a_{N_{t}-1}\right)^{T}, \boldsymbol{\tau}=\left(\tau_{0} \ldots, \tau_{N_{t}-1}\right)$, and

$$
\boldsymbol{P}(\boldsymbol{\tau})=\left(\begin{array}{ccc}
p\left(0-\tau_{0}\right) & \ldots & p\left(0-\tau_{N_{t}-1}\right) \\
p\left(T_{s}-\tau_{0}\right) & \ldots & p\left(T_{s}-\tau_{N_{t}-1}\right) \\
\vdots & \ldots & \vdots \\
p\left((L-1) T_{s}-\tau_{0}\right) & \ldots & p\left((L-1) T_{s}-\tau_{N_{t}-1}\right)
\end{array}\right) .
$$

Equation (4) can be rewritten as:

$$
\boldsymbol{y}=\boldsymbol{X} \boldsymbol{P}(\boldsymbol{\tau}) \boldsymbol{a}+\boldsymbol{w}=\boldsymbol{S}(\tau) \boldsymbol{a}+\boldsymbol{w}
$$

with $\boldsymbol{S}(\boldsymbol{\tau})=\boldsymbol{X} \boldsymbol{P}(\boldsymbol{\tau})$. In the following, for ease of notation, we omit the dependence on $\tau$ and we use $\boldsymbol{P}$ and $\boldsymbol{S}$ instead of $\boldsymbol{P}(\boldsymbol{\tau})$ and $\boldsymbol{S}(\boldsymbol{\tau})$. We also use $\hat{\boldsymbol{P}}$ and $\hat{\boldsymbol{S}}$ instead of $\boldsymbol{P}(\hat{\boldsymbol{\tau}})$ and $S(\hat{\tau})$.

To evaluate the CRB $\boldsymbol{C R} \boldsymbol{B}_{S}(\boldsymbol{h})$ of this estimator, we introduce two matrices $\dot{\boldsymbol{S}}$ and $\dot{\boldsymbol{P}}$ calculated as follows:

$$
\begin{gathered}
\{\dot{\boldsymbol{S}}\}_{k p}=\frac{\partial}{\partial \tau_{p}} \sum_{l=0}^{L-1} x((L-1)+k-l) p\left(l T_{s}-\tau_{p}\right) \\
1 \leq k \leq\left(N_{p}-L+1\right) ; 1 \leq p \leq N_{t}, \\
\{\dot{\boldsymbol{P}}\}_{l p}=\frac{\partial}{\partial \tau_{p}} p\left(l T_{s}-\tau_{p}\right) \quad 1 \leq l \leq L ; 1 \leq p \leq N_{t} .
\end{gathered}
$$

Referring to [2] and considering $\boldsymbol{\xi}=(\boldsymbol{a}, \boldsymbol{\tau})^{T}$, we can determine jointly the values of the attenuations $\boldsymbol{a}$ and the delays $\boldsymbol{\tau}$. From the estimated vector $\hat{\boldsymbol{\xi}}=(\hat{\boldsymbol{a}}, \hat{\boldsymbol{\tau}})^{T}$, we can deduce the value of the estimated $\hat{\boldsymbol{h}}$.

The log-likelihood function for $\boldsymbol{\xi}=(\boldsymbol{a}, \boldsymbol{\tau})^{T}$ given the observation $\boldsymbol{y}$ is:

$\log p(\boldsymbol{y}, \boldsymbol{\xi})=-\left(\frac{N_{p}-L+1}{2}\right) \log \left(\pi \sigma_{w}^{2}\right)-\frac{1}{\sigma_{w}^{2}}\|\boldsymbol{y}-\boldsymbol{S} \boldsymbol{a}\|_{2}^{2}$

The complex Fisher information matrix for the parameter vector as $\boldsymbol{\xi}=(\boldsymbol{a}, \boldsymbol{\tau})^{T}$ is:

$$
\begin{aligned}
& \boldsymbol{F}(\boldsymbol{\xi})=\mathbb{E}\left\{\boldsymbol{\nabla}_{\boldsymbol{h}} \log p(\boldsymbol{y}, \boldsymbol{\xi})\left[\boldsymbol{\nabla}_{\boldsymbol{h}} \log p(\boldsymbol{y}, \boldsymbol{\xi})\right]^{H}\right\} \\
& =\frac{1}{\sigma_{w}^{2}}\left(\begin{array}{cc}
\boldsymbol{S}^{H} \boldsymbol{S} & \boldsymbol{S}^{H} \dot{\boldsymbol{B}} \\
\dot{\boldsymbol{B}}^{H} \boldsymbol{S} & 2 \Re\left\{\dot{\boldsymbol{B}}^{H} \dot{\boldsymbol{B}}\right\}
\end{array}\right)
\end{aligned}
$$


where $\Re\{()$.$\} stands for the real part of (.) and, \dot{B}=$ $\dot{\boldsymbol{S}} \operatorname{diag}(\boldsymbol{a})$, with $\operatorname{diag}(\boldsymbol{x})$ is a matrix which puts $\boldsymbol{x}$ on the main diagonal.

The CRB of this structured LS estimator (S-LSE) of $\boldsymbol{h}$ is calculated as follows [2]:

$$
\boldsymbol{C R} \boldsymbol{B}_{S}(\boldsymbol{h})=\boldsymbol{J F}(\boldsymbol{\xi})^{-1} \boldsymbol{J}^{H} \leq \boldsymbol{C R} \boldsymbol{B}_{\boldsymbol{U}}(\boldsymbol{h}) .
$$

Considering $\dot{\boldsymbol{A}}=\dot{\boldsymbol{P}} \operatorname{diag}(\boldsymbol{a}), \boldsymbol{J}=[\boldsymbol{P}, \dot{\boldsymbol{A}}]$ denotes the Jacobian corresponding to equation (8).

Using the formula for the inverse of a block matrix $\boldsymbol{F}(\boldsymbol{\xi})$ as well as the matrix inversion lemma, after some algebra we obtain:

$$
\boldsymbol{J} \boldsymbol{F}(\boldsymbol{\xi})^{-1} \boldsymbol{J}^{H}=\sigma_{w}^{2} \boldsymbol{P}\left(\boldsymbol{S}^{H} \boldsymbol{S}\right)^{-1} \boldsymbol{P}^{H}+\boldsymbol{\Phi}
$$

where $\Phi$ is a term that depends on the CRB of the estimation of the propagation delay $\boldsymbol{\tau}$, denoted $C R B(\boldsymbol{\tau})$. Finally, $\boldsymbol{\Phi}$ is determined as follows:

$$
\begin{aligned}
\boldsymbol{\Phi}= & \boldsymbol{P}\left(\boldsymbol{S}^{\dagger} \dot{\boldsymbol{B}}\right) C R B(\boldsymbol{\tau})\left(\boldsymbol{S}^{\dagger} \dot{\boldsymbol{B}}\right)^{H} \boldsymbol{P}^{H}+\dot{\boldsymbol{A}} C R B(\boldsymbol{\tau}) \dot{\boldsymbol{A}}^{H} \\
& +\dot{\boldsymbol{A}} C R B(\boldsymbol{\tau})\left(\boldsymbol{P} \boldsymbol{S}^{\dagger} \dot{\boldsymbol{B}}\right)^{H}+\boldsymbol{P} \boldsymbol{S}^{\dagger} \dot{\boldsymbol{B}} C R B(\boldsymbol{\tau}) \dot{\boldsymbol{A}}^{H}
\end{aligned}
$$

where, $\boldsymbol{x}^{\dagger}=\left(\boldsymbol{x}^{H} \boldsymbol{x}\right)^{-1} \boldsymbol{x}^{H}$, is a generalized inverse matrix of $\boldsymbol{x}$ (pseudo-inverse).

Given that $C R B(\tau)$ is the second term diagonal of the matrix $\boldsymbol{F}(\boldsymbol{\xi})^{-1}$, by identification we have the following expression [2]:

$$
C R B(\boldsymbol{\tau})=\sigma_{w}^{2}\left[2 \Re\left\{\dot{\boldsymbol{B}}^{H} \dot{\boldsymbol{B}}\right\}-\dot{\boldsymbol{B}}^{H} \boldsymbol{S} \boldsymbol{S}^{\dagger} \dot{\boldsymbol{B}}\right]^{-1} .
$$

Since the impulse response can be expressed as a function of the parameters via (8), the MSE for the estimation of $\hat{\boldsymbol{h}}_{\boldsymbol{S}-\boldsymbol{L} \boldsymbol{S} \text {, }}$ a LS estimator based on the multi-path parametric model, is lower bounded by:

$$
\begin{aligned}
& \operatorname{MSE}\left(\hat{h}_{S-L S}\right) \geq \operatorname{tr}\left\{C \boldsymbol{C R B} B_{S}(h)\right\} \\
& \geq \sigma_{w}^{2} \operatorname{tr}\left\{\boldsymbol{P}\left(\boldsymbol{S}^{H} \boldsymbol{S}\right)^{-1} \boldsymbol{P}^{H}\right\}
\end{aligned}
$$

Indeed, equation (14) clearly shows that the amplitude estimation is penalized when the delays are not known and it is coupled with the delay error via the additional contribution represented by the term $\Phi$.

Let us now consider the particular interesting case when the vector $\tau$ is known (based on geo-localization and a geometrical model of the scene for example). We get:

$$
C R B(\boldsymbol{\tau})=\mathbf{0} ; \boldsymbol{\Phi}=\mathbf{0}
$$

Thus, knowing the value of $\tau$, the channel estimation is reduced to the estimation of the vector of the fading coefficients $\hat{\boldsymbol{a}}$ which is given by

$$
\left\{\begin{aligned}
\hat{\boldsymbol{a}}_{\boldsymbol{L} \boldsymbol{S}} & =\left(\boldsymbol{S}^{H} \boldsymbol{S}\right)^{-1} \boldsymbol{S}^{H} \boldsymbol{y} \\
\hat{\boldsymbol{h}}_{\boldsymbol{S}-\boldsymbol{L} \boldsymbol{S}} & =\boldsymbol{P} \hat{\boldsymbol{a}}_{\boldsymbol{L} \boldsymbol{S}}
\end{aligned}\right.
$$

In the presence of AWGN noise, the MSE of the estimated vector $\hat{\boldsymbol{h}}_{\boldsymbol{S}-\boldsymbol{L} \boldsymbol{S}}$ is given by:

$$
\boldsymbol{M S E}\left(\hat{\boldsymbol{h}}_{\boldsymbol{S}-\boldsymbol{L} \boldsymbol{S}}\right)=\sigma_{w}^{2} \operatorname{tr}\left\{\boldsymbol{P}\left(\boldsymbol{S}^{H} \boldsymbol{S}\right)^{-1} \boldsymbol{P}^{H}\right\}
$$

In the following, we further assume that we have an ideal training sequence satisfying

$$
\frac{1}{N_{p}-L+1} \boldsymbol{X}^{H} \boldsymbol{X}=\sigma_{p}^{2} \boldsymbol{I}_{L \times L}
$$

By applying the assumption (19) and after some matrix algebra, we obtain:

$$
\begin{aligned}
& \boldsymbol{M S E}\left(\hat{\boldsymbol{h}}_{\boldsymbol{U}-\boldsymbol{L} \boldsymbol{S}}\right)=\operatorname{tr}\left\{\boldsymbol{C R} \boldsymbol{B}_{\boldsymbol{U}}(\boldsymbol{h})\right\}=\frac{\sigma_{w}^{2}}{\sigma_{p}^{2}\left(N_{p}-L+1\right)} L \\
& \boldsymbol{M S} \boldsymbol{E}\left(\hat{\boldsymbol{h}}_{\boldsymbol{S}-\boldsymbol{L} \boldsymbol{S}}\right)=\operatorname{tr}\left\{\boldsymbol{C R} \boldsymbol{B}_{\boldsymbol{S}}(\boldsymbol{h})\right\} \\
& =\frac{\sigma_{w}^{2}}{\sigma_{p}^{2}\left(N_{p}-L+1\right)} \operatorname{tr}\left\{\boldsymbol{P}\left(\boldsymbol{P}^{H} \boldsymbol{P}\right)^{-1} \boldsymbol{P}^{H}\right\}=\frac{\sigma_{w}^{2}}{\sigma_{p}^{2}\left(N_{p}-L+1\right)} N_{t}
\end{aligned}
$$

As a consequence, with the same number of pilot symbols and the same value of signal-to-noise (SNR) ratio $S N R=\sigma_{p}^{2} / \sigma_{w}^{2}$, the structured and unstructured estimators can reach two different values for the MSE and it can be shown that

$$
\operatorname{MSE}\left(\hat{\boldsymbol{h}}_{S-L S}\right) \leq M S E\left(\hat{\boldsymbol{h}}_{U-L S}\right) .
$$

The performance gain in terms of MSE given by the structured estimator is given by:

$$
G_{d B}=10 \log _{10}\left(\frac{\boldsymbol{M S E}\left(\hat{\boldsymbol{h}}_{U-L S}\right)}{\boldsymbol{M S E}\left(\hat{\boldsymbol{h}}_{S-L S}\right)}\right)=10 \log _{10}\left(\frac{L}{N_{t}}\right) .
$$

According to the above expression, the gain provided by the SLSE estimation with respect to the U-LSE estimation becomes more important if the number of paths $N_{t}$ is very small compared to the channel memory $L$. The preceding study will be used for the derivation of an efficient estimation method for aeronautical communications via a satellite channel.

\section{APPLICATION TO AERONAUTICAL COMMUNICATIONS VIA A SATELLITE LINK}

\section{A. Channel model}

The aeronautical satellite channel, proposed in [5], is characterized by a strong line of sight (LOS) component that is present most of the time. Depending on the type of ground and the geometry of the scene, multiple delayed reflections from the ground arrive at the aircraft with a given attenuation compared to the LOS component (Fig.1). The delay spread associated to these reflexions is very small compared to the signal period.

The direct signal from the satellite, can also undergo reflections on the surface of fuselage aircraft before being intercepted by the receiver omnidirectional antenna. These local scatterers have very small delays with respect to the considered signal period. Thus, the line of sight (LOS) signal is the sum of a direct signal and a non frequency selective Rayleigh fading process due to the scatterers on the fuselage. 


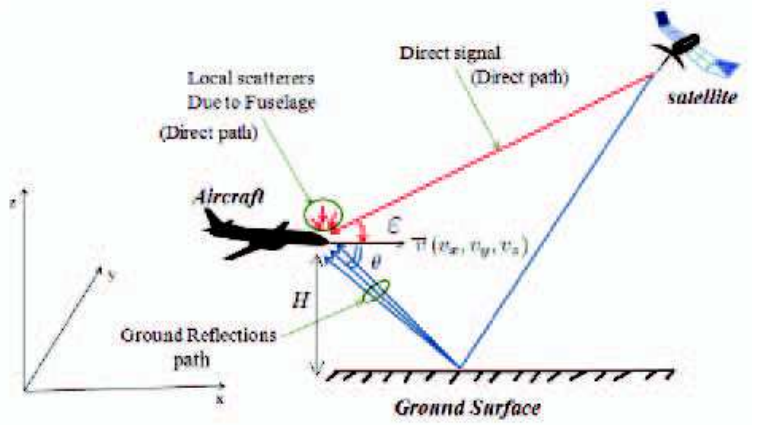

Fig. 1: Geometry illustration of the aeronautical communication channel.

In conclusion, the aeronautical channel is described as a multipath channel consisting essentially of two main paths, a direct path (noted LOS) between the satellite and the aircraft to which is added a reflection path on the ground surface (noted GR for Ground Reflexion) [5]. The LOS path has an attenuation coefficient $a_{L O S}$ that follows a Rice distribution with a Rice factor $\left[k_{L O S}\right]_{d B} \approx 14 d B$ [6]. The direct path is considered as the reference path in terms of time. The GR path representing the reflection on the ground is delayed by $\tau_{G R}$ and affected by an attenuation $a_{G R}$ which follows a Rayleigh distribution [6]. The power of each path $\mathbb{E}\left\{\left|a_{L O S}\right|^{2}\right\}$ and $\mathbb{E}\left\{\left|a_{G R}\right|^{2}\right\}$ is deduced by knowing the signal-to-multipath power ratio, denoted $C / M$ given by:

$$
\left\{\begin{array}{c}
C / M=10 \log _{10}\left(\frac{\mathbb{E}\left\{\left|a_{L O S}\right|^{2}\right\}}{\mathbb{E}\left\{\left|a_{G R}\right|^{2}\right\}}\right) \\
\text { with: } \mathbb{E}\left\{\left|a_{G R}\right|^{2}\right\}+\mathbb{E}\left\{\left|a_{G R}\right|^{2}\right\}=1
\end{array}\right.
$$

Taking into account the geometrical properties of the scene, it is possible to estimate $\tau_{G R}$ if some a priori information on the position of the aircraft is available. This can be achieved by using some geolocation techniques. An estimate of $\tau_{G R}$ can be calculated from the knowledge of the elevation angle $\varepsilon$ between the aircraft and a geostationary satellite obtained by GPS measurements [7]:

$$
\begin{aligned}
\hat{\tau}_{G R} & =\frac{2 H \sin (\widehat{\varepsilon})}{c} \text { where } \\
\sin (\widehat{\varepsilon}) & =\frac{\cos \left(\hat{\theta}_{1}\right) \cos \left(\phi_{2}-\hat{\phi}_{1}\right)-\frac{R+H}{R+D}}{\sqrt{1-2 \cos \left(\hat{\theta}_{1}\right) \cos \left(\phi_{2}-\hat{\phi}_{1}\right) \frac{R+H}{R+D}+\left(\frac{R+H}{R+D}\right)^{2}}}
\end{aligned}
$$

with $\theta_{1}, \phi_{1}$ and $H$ respectively denote the latitude, longitude and altitude of the aircraft, $\phi_{1}$ and $D$ denote the longitude and the altitude of a geostationary satellite. The latitude of the geostationary satellite is approximately zero, $c=310^{8} \mathrm{~m} . \mathrm{s}^{-1}$ is the speed of light and $R=6378,14 \mathrm{~km}$ is the radius of the earth.

By studying the distribution function of the relative error value of $\tau_{G R}$ based on GPS measurements, it can be shown that the percentage of error does not exceed $4,510^{-3} \%$ (See Fig. 2).

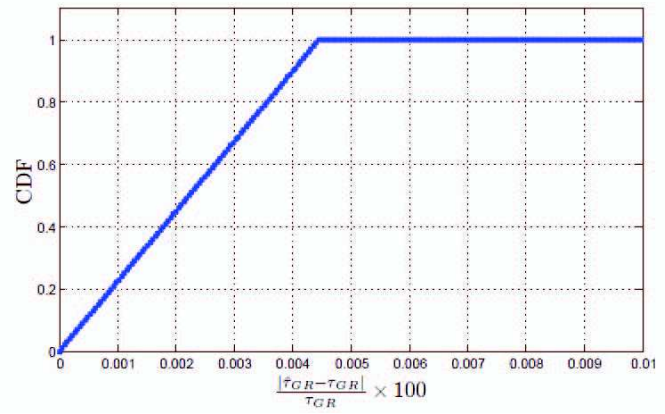

Fig. 2: CDF of the estimation error $\Delta \tau=\hat{\tau}_{G R}-\tau_{G R}$.

Therefore the estimation error for $\tau_{G R}$ can be neglected and the assumption made in the previous section is valid (ie: $\left.\hat{\tau}_{G R}=\tau_{G R}\right)$.

We now consider two different approaches for the estimation of the aeronautical channel that exploit its main characteristics.

\section{B. Channel Estimation using a Multipath Parametric Model}

The aeronautical channel can be regarded as a combination of two waveforms, $a_{L O S} p(t)$ and $a_{G R} p\left(t-\tau_{G R}\right)$ (See Fig. 3).

$$
h(t)=a_{L O S} p(t)+a_{G R} p\left(t-\tau_{G R}\right)
$$

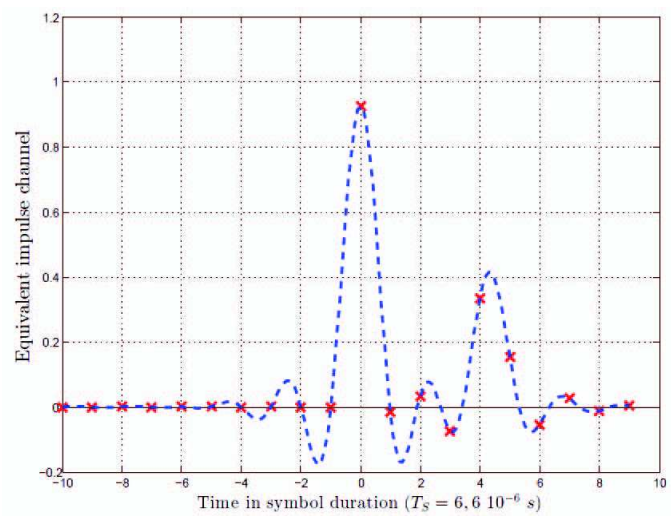

Fig. 3: An illustration for measured impulse responses for airplane approaching the ground.

The equivalent channel impulse response depends on two parameters: the delay $\tau_{G R}$ and the attenuation vector $\left(a_{L O S}, a_{G R}\right)$. The knowledge of these two parameters enables us to deduce the value of the channel impulse response. This is a multi-path parametric model, and so we can apply the estimation method as described in II-C.

In this case, the parameters $\boldsymbol{\tau}$ and $\boldsymbol{a}$ can be written as $\boldsymbol{\tau}=$ $\left(0, \tau_{G R}\right)^{T}$ and $\boldsymbol{a}=\left(a_{L O S}, a_{G R}\right)^{T}$. In addition, by geometrical considerations, we can determine with great accuracy the value $\tau_{G R}$. This a priori information can greatly reduce the variance of the estimator. In fact, the estimation of $\boldsymbol{h}$ is only dependent on the estimation of the parameter $\boldsymbol{a}$ which reduces to:

$$
\left\{\begin{aligned}
\hat{\boldsymbol{a}}_{\boldsymbol{L} \boldsymbol{S}} & =\left(\boldsymbol{S}^{H} \boldsymbol{S}\right)^{-1} \boldsymbol{S}^{H} \boldsymbol{y} \quad \text { with, } \boldsymbol{S}=\boldsymbol{X} \boldsymbol{P}\left(\tau_{G R}\right) \\
\hat{\boldsymbol{h}} & =\boldsymbol{P} \hat{\boldsymbol{a}}_{\boldsymbol{L} \boldsymbol{S}}
\end{aligned}\right.
$$


where

$$
\boldsymbol{P}\left(\tau_{G R}\right)=\left(\begin{array}{cc}
p(0) & p\left(0-\tau_{G R}\right) \\
p\left(T_{s}\right) & p\left(T_{s}-\tau_{G R}\right) \\
\vdots & \vdots \\
p\left((L-1) T_{s}\right) & p\left((L-1) T_{s}-\tau_{G R}\right)
\end{array}\right)
$$

Applying this method, an estimation gain with respect the use of a classical LS estimator U-LSE can be achieved:

$$
G_{d B}=10 \log _{10}\left(\frac{L}{2}\right)
$$

\section{Channel Estimation as a Sparse Estimation Problem}

To some extent, the discrete impulse response of an aeronautical channel can be considered as sparse [8] if some taps are neglected. Indeed, there are two or three powerful taps and other low power taps can be neglected (See Fig. 3). This can be efficiently used for low complexity equalization [8] [9]. We now show how it can be used to derive another method for channel estimation in the aeronautical context.

To build the corresponding sparse channel $\tilde{\boldsymbol{h}}$ from the aeronautical channel impulse $\boldsymbol{h}$, we neglect from $\boldsymbol{h}$ the lowpower paths. To this end, one chooses a power ratio threshold $s$, normalized to the most powerful path, below which it is assumed that impulse response is negligible and equal to zero as the following:

$$
\tilde{\boldsymbol{h}}(i)= \begin{cases}\boldsymbol{h}(i) & \text { if } \frac{\|\boldsymbol{h}(i)\|_{2}^{2}}{\max _{i}\left\{\|\boldsymbol{h}(i)\|_{2}^{2}\right\}} \geq s \\ 0 & \text { else }\end{cases}
$$

Starting with this assumption, we can use some estimation methods in the literature [10] [11] which exploit the particular form of the channel. Instead of estimating $\boldsymbol{h}$, we will estimate $\tilde{\boldsymbol{h}}$ as a good approximation for $\boldsymbol{h}$.

In the case of the aeronautical channel, we can follow the work by Carbonelli and all [4]. The authors are interested in joint estimation of non-zero positions and the corresponding attenuation coefficients of a sparse multipath channel. For the sparse vector $\tilde{\boldsymbol{h}}$, we associate a binary sparse vector $\boldsymbol{b}$ which detects the non-zero positions obtained as [4]:

$$
\boldsymbol{b}(i)= \begin{cases}1 & \text { if } \tilde{\boldsymbol{h}}(i) \neq 0 \\ 0 & \text { else }\end{cases}
$$

In this case the vector of the samples received can be written as follows [4]:

$$
\boldsymbol{y}=\boldsymbol{X} \tilde{\boldsymbol{h}}+\boldsymbol{w}=\boldsymbol{X} \operatorname{diag}(\boldsymbol{b}) \boldsymbol{h}+\boldsymbol{w}=\boldsymbol{X} \operatorname{diag}(\boldsymbol{h}) \boldsymbol{b}+\boldsymbol{w}
$$

In the general case where there is no a priori on the vector position of non-zero coefficients $\boldsymbol{b}$, the estimation is done in two steps: first, estimation of vector $\hat{b}$ that contains either 0 or 1 , second, computation of an estimation of $\tilde{\boldsymbol{h}}$ derived from $\hat{b}$ as follows [4]:

$$
\left\{\begin{array}{l}
\hat{\boldsymbol{b}}=\underset{\tilde{\boldsymbol{b}}}{\operatorname{argmax}}\left\{\left\|\boldsymbol{y}-\left[\boldsymbol{X} \operatorname{diag}\left(\boldsymbol{X}^{\dagger} \boldsymbol{y}\right)\right] \tilde{\boldsymbol{b}}\right\|^{2}\right\} \\
\widehat{\tilde{\boldsymbol{h}}}=\left(\hat{\boldsymbol{X}}_{\boldsymbol{b}}^{H} \hat{\boldsymbol{X}}_{\boldsymbol{b}}\right)^{-1} \hat{\boldsymbol{X}}_{\boldsymbol{b}}^{H} \boldsymbol{y} \quad ; \text { where } \hat{\boldsymbol{X}}_{\boldsymbol{b}}=\boldsymbol{X} \operatorname{diag}(\hat{\boldsymbol{b}})
\end{array}\right.
$$

To avoid an exhaustive search to estimate $\boldsymbol{b}$ and reduce the complexity of the problem, we can use for example the Viterbi algorithm [12]. However, in the aeronautical channel case, the complexity can be reduced. If, we assume that we can have an accurate value of the delay $\tau_{G R}$. Consequently, we can accurately identify the positions of non-zero coefficients of the channel considered as sparse (ie: $\hat{b}=\boldsymbol{b}$ ).

Knowing the value of $\boldsymbol{b}$, the estimation of the discrete sparse channel $\widehat{\tilde{h}}$ is given by [4]:

$$
\widehat{\tilde{\boldsymbol{h}}}=\left(\boldsymbol{X}_{\boldsymbol{b}}{ }^{H} \boldsymbol{X}_{\boldsymbol{b}}\right)^{-1} \boldsymbol{X}_{\boldsymbol{b}}{ }^{H} \boldsymbol{y}
$$

In the presence of AWGN noise, the variance of the estimator of sparse channel reaches the Cramer Rao Bound [4]:

$$
\begin{aligned}
\boldsymbol{M S E}(\widehat{\tilde{\boldsymbol{h}}}) & =\operatorname{tr}\left[\sigma_{w}^{2}\left(\boldsymbol{X}_{\boldsymbol{b}}{ }^{H} \boldsymbol{X}_{\boldsymbol{b}}\right)^{\dagger}\right] \\
& =\frac{\sigma_{w}^{2}}{\sigma_{p}^{2}\left(N_{p}-L+1\right)} \operatorname{tr}\{\operatorname{diag}(\boldsymbol{b})\}
\end{aligned}
$$

Considering the channel as sparse is not really true. By doing so, we introduced a bias in the calculation of the variance, due to a mismatch of the channel model.

$$
\boldsymbol{M S E}(\hat{\boldsymbol{h}})=\operatorname{MSE}(\widehat{\tilde{\boldsymbol{h}}})+\mathbb{E}\left\{\|\boldsymbol{h}-\tilde{\boldsymbol{h}}\|_{2}^{2}\right\}
$$

Assuming $M=\operatorname{tr}\{\operatorname{diag}(\boldsymbol{b})\}$ is the number of the nonzero coefficients in $\tilde{\boldsymbol{h}}$, this method gives an estimation gain, compared with the use of a LS estimator U-LSE given by:

$$
\begin{aligned}
G_{d B} & =10 \log _{10}\left(\frac{\boldsymbol{M S E}\left(\hat{\boldsymbol{h}}_{U-L S}\right)}{\boldsymbol{M S E}(\hat{\boldsymbol{h}})}\right) \\
& =-10 \log _{10}\left(\frac{M}{L}+\frac{\mathbb{E}\left\{\|\boldsymbol{h}-\tilde{\boldsymbol{h}}\|_{2}^{2}\right\}}{\boldsymbol{M S E}\left(\hat{\boldsymbol{h}}_{U-L S}\right)}\right)
\end{aligned}
$$

The gain achieved by this method depends on the choice of non-zero coefficients $M$.

Referring to (31), we can prove that the achieved gain is always positive whatever the choice of $M$, i.e:

$$
G_{d B} \geq 0 \quad \forall 1 \leq M \leq L
$$

To conclude, the sparse consideration enables a reduced complexity method. However, it is not as efficient as the S-LS method that considers the multipath parametric model. This leads to complexity-versus performance trade-off.

\section{Results}

We consider in this section a channel length $L=20$ and a training sequence of length $N=25$. In Figure4, we compare in terms of Mean Square Error (MSE) the two estimation techniques U-LS and S-LS. The Cramer-Rao bounds for both methods $\operatorname{tr}\left(\boldsymbol{C R} \boldsymbol{B}_{U}\right), \operatorname{tr}\left(\boldsymbol{C R} \boldsymbol{B}_{S}\right)$ are also plotted. Since the aeronautical channel has two paths, there is a gain of $10 \mathrm{~dB}$ provided by the S-LSE technique compared to the U-LSE technique. 


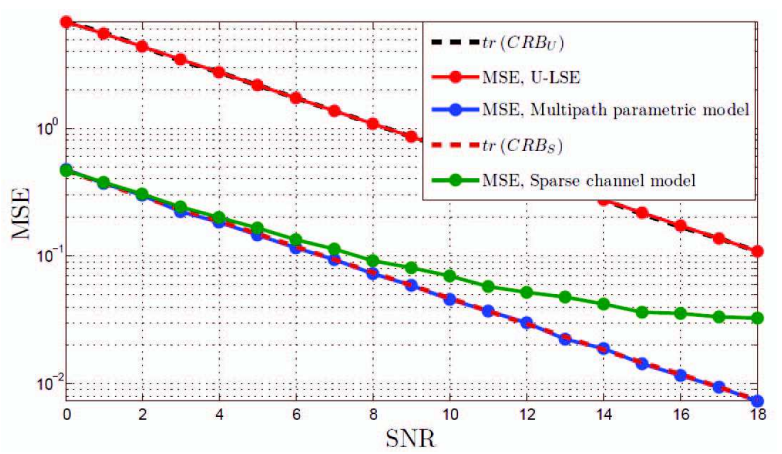

Fig. 4: MSE, CRB-U and CRB-S for the unstructured and the structured LS estimator.

Fig.5 and Fig.6 present the performance of BPSK (Binary Phase Shift Keying) modulation in terms of bit error rate (BER) for the uncoded case and the coded case, respectively. For the coded case we use ARJA (accumulate-repeat jagged accumulate) protograph based Low Density Parity Check (LDPC) code of rate $R=4 / 5$ with 50 iterations as channel coding.

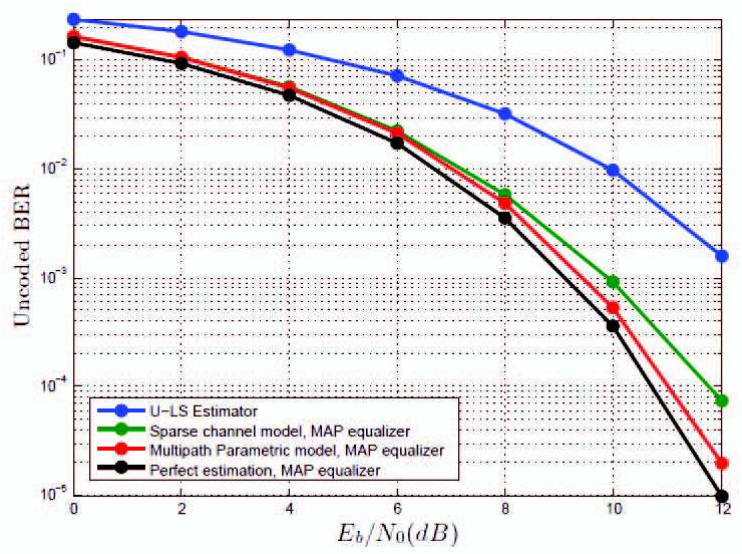

Fig. 5: Uncoded BER for the U-LS and the S-LS estimators.

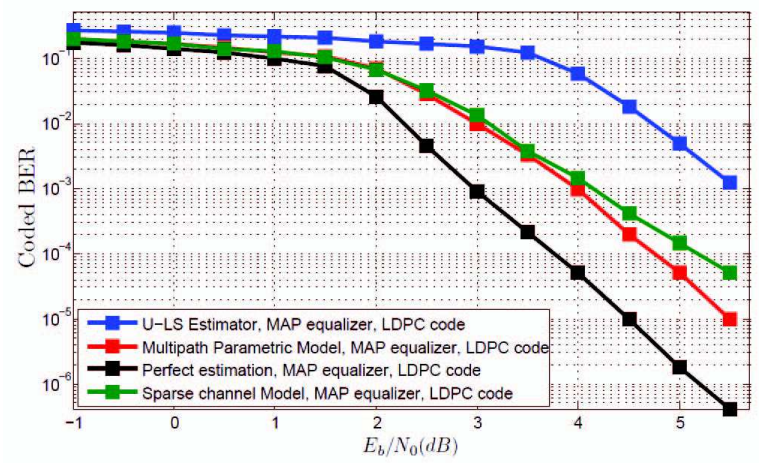

Fig. 6: Coded BER for the U-LS and the S-LS estimators.

We only consider serial channel equalization and channel decoding. At the reception we adopt an equalization scheme according to the MAP criterion (Maximum a priori) based on channel estimation. For the two figures, the blue curve is relative to equalization using the U-LSE method for estimating the discrete equivalent channel. The red and the green curves are relative to equalization using the S-LSE channel estimation method considering the parametric multipath model and the sparse parametric model, respectively. The black curve represents the equalization based on a perfect knowledge of the discrete channel response.

Results show that the S-LSE technique is better suited to the MAP equalization and offers a significant gain in terms of BER compared to the classical U-LS method. Furthermore, the results show that the estimation technique using a parametric Multipath model has the same performance than the technique that uses the sparse consideration in the low SNR region. However, for high SNR, we can see that the first technique has better performances than the second one in terms of MSE and BER.

\section{CONCLUSION}

In this paper, we proposed some efficient channel estimation methods for aeronautical communications via a satellite link. The proposed methods exploit both the particular form of the channel impulse response and a priori knowledge of certain parameters that can be determined by geometrical considerations. This reduces the number of variables to be estimated and provides better performances compared to algorithms that do not exploit this knowledge. Future works will investigate on these methods for turbo-equalization [13] to reduce the impact of imperfect channel estimation. Moreover, impact on linear equalization methods will also be considered.

\section{REFERENCES}

[1] M. Siala, "Maximum a posteriori fast fading channel estimation based exclusively on pilot symbols," Annales Des Télécommunications, pp. 569-586, 2001.

[2] M. Sharp and A. Scaglione, "Estimation of sparse multipath channels," IEEE MILCOM Conf., vol. 57, no. 2, pp. 64-68, 2009.

[3] J. Soubielle, I. Fijalkow, P. Duvaut, and a. Bibaut, "GPS positioning in a multipath environment," IEEE Trans. on Signal Processing,, vol. 50, no. 1, pp. 141-150, 2002.

[4] C. Carbonelli, S. Vedantam, and U. Mitra, "Sparse channel estimation with zero tap detection," IEEE Trans. on Wireless Comm., vol. 6, no. 5, pp. 1743-1753, 2007

[5] M. Richharia, N. Kaluvala, P. Fines, M. Álvarez-Díaz, and A. Jahn, "Aeronautical channel model for broadband l-band satellite communication," in AIAA International Communications Satellite Systems Conference (ICSSC), Rome, Italy, September 25-28 2005.

[6] P. Bello, "Aeronautical Channel Characterization," IEEE Transactions on Communications, vol. 21, no. 5, pp. 548-563, May 1973.

[7] T. Kos, I. Markezic, and J. Pokrajcic, "Effects of multipath reception on GPS positioning performance," Elmar, 2010 Proceedings, no. 2, pp. 15-17, September 2010.

[8] B. Raddadi, N. Thomas, C. Poulliat, M.-1. Boucheret, and B. Gadat, "On an efficient equalization structure for aeronautical communications via a satellite link ." IEEE STWIMOB, pp. 396-401, October 2014.

[9] J. Park and S. Gelfand, "Sparse map equalizers for turbo equalizations," vol. 2, pp. 762-766 Vol. 2, May 2005.

[10] S. F. Cotter and B. D. Rao, "Sparse channel estimation via matching pursuit with application to equalization," IEEE Transactions on Communications, vol. 50, no. 3, pp. 374-377, 2002.

[11] N. C. Sampling, "Asymptotic Achievability of the Cramer-Rao Bound for Noisy Compressive Sampling," Complexity, vol. 57, no. 3, pp. 12331236, 2009.

[12] N. Benvenuto and R. Marchesani, "The viterbi algorithm for sparse channels," Communications, IEEE Transactions on, vol. 44, no. 3, pp. 287-289, Mar 1996.

[13] J. Park and S. Gelfand, "Turbo equalizations for sparse channels," vol. 4, pp. 2301-2306 Vol.4, March 2004. 